\title{
What Went Where When? Representing Animal Movements as Simple Darwin Core Occurrences
}

\author{
Peggy Newman ${ }^{\ddagger}$, Holger Dettki§, Lee Belbinl \\ ‡ Atlas of Living Australia, Canberra, Australia \\ § Swedish University of Agricultural Sciences, Umeå, Sweden \\ | Blatant Fabrications Pty Ltd, Carlton, Australia
}

Corresponding author: Peggy Newman (peggy.newman@csiro.au)

Received: 10 Apr 2018 | Published: 21 May 2018

Citation: Newman P, Dettki H, Belbin L (2018) What Went Where When? Representing Animal Movements as

Simple Darwin Core Occurrences. Biodiversity Information Science and Standards 2: e25664.

https://doi.org/10.3897/biss.2.25664

\section{Abstract}

To usefully describe sensor deployments on animals is a major challenge for advocates of data standards. Bio-logging studies also need to be documented in a standard manner to facilitate discovery and determine relevance? For systems aggregating biodiversity occurrence records, the use of the Darwin Core standard (Wieczorek et al. 2012) to express species occurrences is near ubiquitous. Bio-logging studies are universally multiple instances of species occurrences that output high quality spatial and temporal data recorded by specialists.

There are a lot of benefits to summarising these studies by means of a single, flat file record. Simple Darwin Core offers the ability to do this by representing the multiple occurrences as a date range in dwc:eventDate and a footprint polygon using dwc:footprintWKT for the area covered by the track. By also uniformly describing the species, the dwc:basisOfRecord as Machine Observation, and a controlled vocabulary to describe the type of bio-logging data, systems could offer an effective means of querying tracking data. It's important to look to other data standards initiatives relevant to bio-logging to ensure common usage of Darwin Core terms.

The Atlas of Living Australia is using an implementation of Simple Darwin Core to represent data from the bio-logging platform ZoaTrack as occurrence data to make it 
discoverable via location or species-based searches. Other initiatives, for example Swedish LifeWatch follow a similar approach to represent data from the Wireless Remote Animal Monitoring (WRAM) Scandinavian bio-logging infrastructure. With endorsement from the community, the implementation could be useful as a type of metadata catalogue record, opening it for usage in application programmer interface (API) development and thus enabling machine interoperability between systems and users. In short, bio-logging systems and practitioners would be able to easily discover relevant studies by searching by location and/or species.

\section{Keywords}

Bio-logging standards, occurrence, Simple Darwin Core, tracking, tagging, interoperability, animal movement, footprint

\section{Presenting author}

Peggy Newman

\section{References}

- Wieczorek J, Bloom D, Guralnick R, Blum S, Döring M, Giovanni R, Robertson T, Vieglais D (2012) Darwin Core: An Evolving Community-Developed Biodiversity Data Standard. PLoS ONE 7 (1): e29715. https://doi.org/10.1371/journal.pone.0029715 